\title{
Bistatic human micro-Doppler signatures for classification of indoor activities
}

\author{
Francesco Fioranelli \\ School of Engineering \\ University of Glasgow \\ University Avenue, G12 8QQ, Glasgow, UK
}

\author{
Matthew Ritchie, Hugh Griffiths \\ Department of Electronic and Electrical Engineering \\ University College London \\ Torrington Place, WC1E 7JE, London, UK
}

\begin{abstract}
This paper presents the analysis of human microDoppler signatures collected by a bistatic radar system to classify different indoor activities. Tools for automatic classification of different activities will enable the implementation and deployment of systems for monitoring life patterns of people and identifying fall events or anomalies which may be related to early signs of deteriorating physical health or cognitive capabilities. The preliminary results presented here show that the information within the micro-Doppler signatures can be successfully exploited for automatic classification, with accuracy up to $98 \%$, and that the multi-perspective view on the target provided by bistatic data can contribute to enhance the overall system performance.
\end{abstract}

Keywords- bistatic radar, micro-Doppler, feature extraction and classification, machine learning

\section{INTRODUCTION}

Radar micro-Doppler signatures are defined as the additional frequency modulations on top of the main Doppler shift of moving targets, and these modulations are related to vibrating or rotating parts of vehicles and aircraft (e.g. the tracks of tanks or the blades of helicopters), as well as to swinging limbs and body parts for humans [1-2]. These signatures have been used in recent years as a source of information to perform the identification and classification of actions for a variety of applications in different contexts. These include discrimination of humans vs animals and vehicles, recognition of different activities performed by humans, identification of specific individuals based on their walking or running gait [3-8].

The use of radar micro-Doppler signatures has been also reported in the context of ambient assisted living, fall detection, and general monitoring of the life patterns and conditions of people, especially elderly or vulnerable people affected by physical or cognitive impairments [9]. Radar systems can provide accurate persistent monitoring capabilities without the privacy concerns associated with camera systems, or requiring the patients to have to wear motion sensor devices or to modify their normal behavior. Different research articles in the literature have discussed aspects such as the study of timefrequency distributions in order to characterize walking with assistive devices like canes, various features to characterize common indoor movements, monitoring of patterns of life and vitality as well as detecting significant fall events when caring for the elderly [9-14]. One of the main issue in using radar
micro-Doppler signatures for classification is their dependence on the cosine of the aspect angle between the target trajectory and the radar line-of-sight. For unfavorable angles close to $90^{\circ}$ this can drastically reduce the classification accuracy [4,15]. Bistatic and multistatic radar systems have been proposed as an effective solution to this issue, as a collection of nodes can be deployed to provide favorable geometry with respect to the targets of interest, for at least a subset of nodes. However, experimental results involving actual bistatic or multistatic radar systems are rather limited, as their development and operations are more challenging than conventional monostatic systems. The issue of providing a unique phase reference to synchronize all the nodes is particularly challenging, increasing the hardware complexity of the whole system [16]

This paper presents the preliminary analysis of radar microDoppler signatures for indoor human activities and investigates simple features and algorithms to perform automatic classification. The data were collected using a single channel radar. First a series of measurements were taken using a colocated transmit/receive antenna monostatic setup, and then these were repeated with a spatially separated receiver for the bistatic equivalent results. It was unfortunately not possible to record both monostatic and bistatic results simultaneously. For the bistatic setup a bistatic angle of approximately $20^{\circ}$ is obtained with respect to the target position. The same type of activities performed by the same subjects were recorded, although not simultaneously. The analysis shows that simple features and simple classifiers extracted from the microDoppler signatures can provide high classification accuracy, up to $98 \%$ in the most favorable cases. Furthermore, it is shown how different subsets of features at different nodes (monostatic and bistatic) are required to provide maximum accuracy. This opens up wide scope for further work in exploring this 'feature diversity' [17] in human indoor activities classification scenarios, aiming to characterize the effect on the overall accuracy of the many operational parameters.

The remainder of this paper is organized as follows. Section II describes the radar system and the experimental setup. Section III presents the analysis of the data and the results. Section IV finally concludes the paper.

\section{RADAR SYSTEM AND EXPERIMENTAL SETUP}

The data were collected by an FMCW off-the-shelf radar sensor operating at $5.8 \mathrm{GHz}$ (C-band). Parameters of interest 
were bandwidth and duration of the linear chirp $400 \mathrm{MHz}$ and $1 \mathrm{~ms}$ respectively, the resulting PRF $1 \mathrm{kHz}$ (sufficient to include the whole human micro-Doppler signature within the unambiguous Doppler region), and transmitted power of approximately $+19 \mathrm{dBm}$. Two directional Yagi antennas were used to collect the data in vertical polarization, with $17 \mathrm{dBi}$ gain and $24^{\circ} \times 24^{\circ}$ beam-width.

Figure 1 shows a sketch view of the experimental setup. These measurements were performed in a typical indoor environment, at the School of Engineering at the University of Glasgow. The room contained pieces of office furniture, namely a large meeting table, a couple of smaller tables, and chairs, located around the subject performing the different movements. Hence it represented a real-world cluttered environment which such a sensor would be required to operate. The radar system was placed near a corner of the room, with the subjects performing the different movements positioned at approximately $4 \mathrm{~m}$ from the radar. In monostatic configuration (in color red in the figure) the transmitter and receiver antennas were co-located and separated by approximately $30 \mathrm{~cm}$, whereas in bistatic configuration (in color green in the figure) the receiver antenna was moved at approximately $1.5 \mathrm{~m}$ from the transmitter and aimed at the target, originating a bistatic angle equal to approximately $20^{\circ}$. It should be noted that the collection of the monostatic and bistatic data was not simultaneous, as the radar sensor has only one receiver channel, but care was taken to perform the movements as consistently as possible for both cases. For the full extended paper further bistatic angles will be included as part of the analysis to further understand the relationship of bistatic angle and classification.

Three subjects took part to this data collection, with the following key body parameters, namely $1.71 \mathrm{~m}$, average body type for person A, $1.89 \mathrm{~m}$, average body type for person B, and $1.84 \mathrm{~m}$, slim body type for person C. Ten recordings of $10 \mathrm{~s}$ each were collected for each activity considered and for each subject, for a total number of datasets equal to 240 (4 movements, 3 subjects, 10 recordings, monostatic and bistatic data). The four activities considered were walking back and forth, sitting and standing on an office chair, bending down to pick up an object from the floor and coming up to stand, and waving with one hand. Sitting and bending were chosen as they might be actions triggering false alarms for systems aimed at fall events detection because of their sudden acceleration component [9]. Repeated waving movements with one hand can be used as a key action to initiate the interaction with smart devices, to look them to a specific user to then detect additional commands (similarly to wave to another human being to ask for attention).

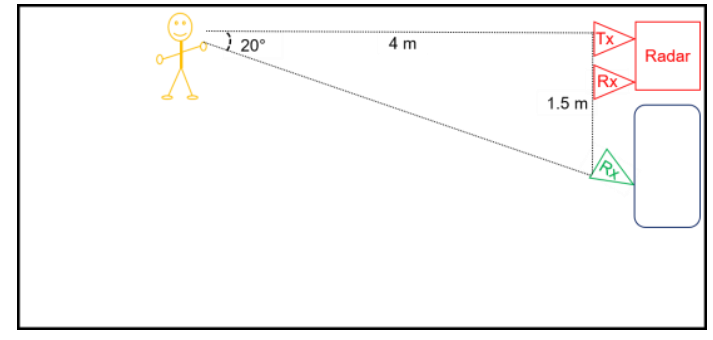

Fig. 1. Plan view of the experimental setup

\section{DATA ANALYSIS AND RESULTS}

The micro-Doppler signatures were extracted from the data by applying the Short Time Fourier Transform (STFT) to generate spectrograms. A Hamming window with 200 samples corresponding to $0.2 \mathrm{~s}$ was used, with $95 \%$ overlap. A notch filter was applied to reduce the contribution of the static clutter around $0 \mathrm{~Hz}$ and highlight the actual signatures. Figure 2 shows examples of spectrograms for the four activities performed by subject $A$. The most evident difference is between the walking signature, which has a dominant positive and negative main Doppler shift caused by the overall motion of the whole body, and the other three signatures, which appear to be centred around $0 \mathrm{~Hz}$ as these were static motions, not involving complete movements of the body. Nevertheless, these static signatures present differences that can be empirically appreciated, suggesting that good automatic classification accuracy can be achieved, provided that relevant features can be developed to extract discriminating information. Figure 3 shows additional examples of spectrograms for the actions of walking and picking up an object performed by person $\mathrm{B}$, for both monostatic and bistatic data. It is interesting to observe the differences in the signatures for the same type of movement, even at the limited bistatic angle of $20^{\circ}$ of this setup. The bistatic signatures appear to have slightly lower Doppler values, e.g. the peaks for the picking up movement reach approximately $75 \mathrm{~Hz}$ for the monostatic case but only around $50 \mathrm{~Hz}$ for the bistatic case. This is compatible with the considered measurement setup, as the movements were performed facing the monostatic node, hence actions such as bending down towards the floor or standing up from the chair are expected to generate the highest Doppler shift in that direction.

Simple features based on the mean and standard deviation of the Doppler centroid and bandwidth of the micro-Doppler signatures were considered to analyze the data. These have been shown to be effective for different classification problems such as personnel recognition [18] and unarmed vs armed personnel classification [19], and have the advantage of not requiring any pre-processing or parameter tuning in the feature extraction algorithm. The Doppler centroid estimates the center of gravity of the micro-Doppler signature, and the Doppler bandwidth calculates the energy extent of the signature around the centroid [17]. These parameters were calculated as in equations (1) and (2), where $S(i, j)$ represents the value of the spectrogram at the $i^{\text {th }}$ Doppler bin and $j^{\text {th }}$ time bin and $f(i)$ is the value of the Doppler frequency at the $\mathrm{i}^{\text {th }} \mathrm{bin}$.

$$
\begin{gathered}
f_{c}(j)=\frac{\sum_{i} f(i) S(i, j)}{\sum_{i} F(i, j)} \\
B_{c}(j)=\sqrt{\frac{\sum_{i}\left(f(i)-f_{c}(j)\right)^{2} S(i, j)}{\sum_{i} S(i, j)}}
\end{gathered}
$$

Each recorded $10 \mathrm{~s}$ dataset was divided into $3 \mathrm{~s}$ blocks and one feature sample extracted from each block, generating 360 samples for each feature for monostatic data and 360 for bistatic data. Figure 4 presents examples of feature space plots for the collected data. The features considered in the $2 \mathrm{D}$ plots 
are the mean and standard deviation of the signature bandwidth, with the addition of the mean value of the signature centroid as $3^{\text {rd }}$ feature in the $3 \mathrm{D}$ plots. These features were selected out of the other different combinations as they empirically provided the best separation between samples belonging to different activities. It should be noted that different colors in figure 4 denote the different activities, whereas the different shapes of the marker (circle, square, and diamond) indicate the different subject. Good separation can be seen between samples of the three static activities in the 2D plots, and the additional feature considered in the 3D plots help separating the walking movement from the others. This appears to be true for both monostatic and bistatic data.
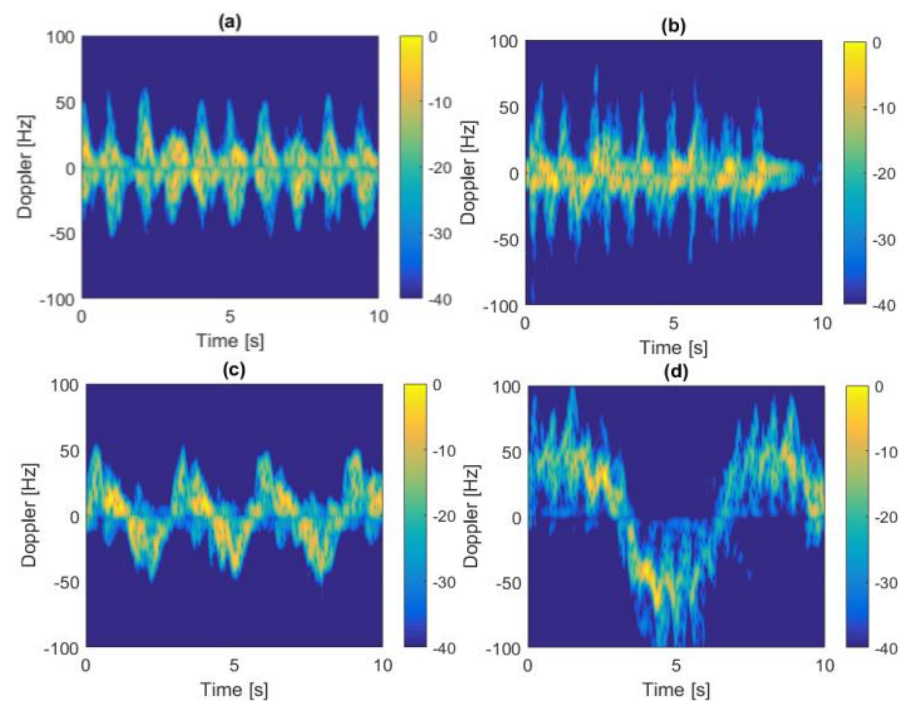

Fig. 2. Spectrograms for different movement performed by the same subject, radar in monostatic configuration: (a) bending and picking up an object from the floor, (b) waving with one hands, (c) sitting and standing up, and (d) walking back and forth
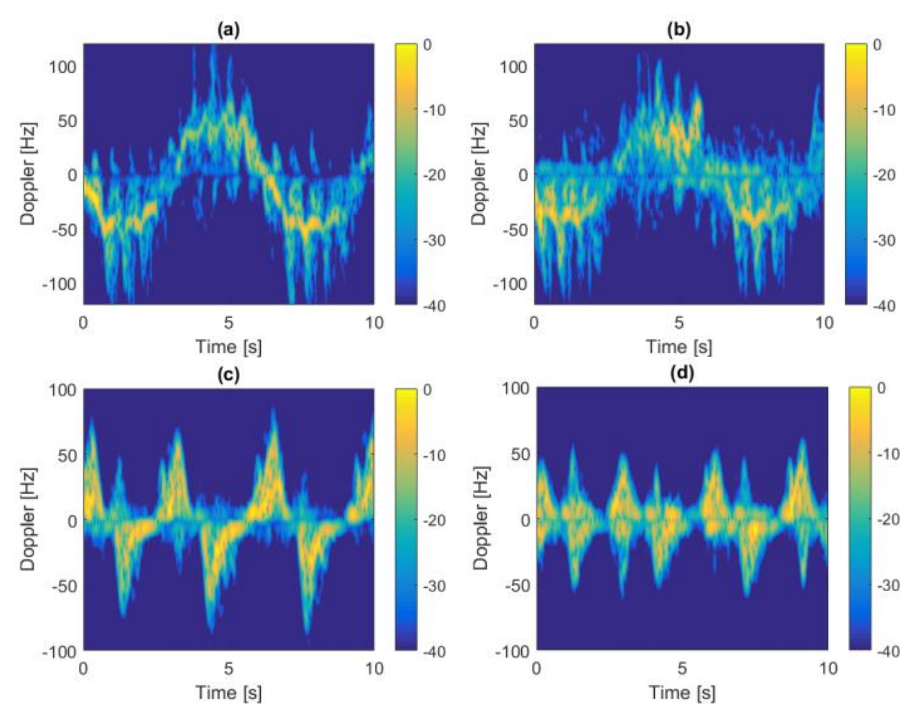

Fig. 3. Comparison of spectrograms in monostatic and bistatic configuration: walking movement monostatic (a) and bistatic (b), and picking up an object monostatic (c) and bistatic (d)
Three different classifiers were used to process the feature samples, namely Binary Tree (BT), Naïve Bayes (NB), and Nearest Neighbor with 3 neighbors ( $3 N N)$. More details on the implementation of these classifiers can be found in [17, 20]. The classifiers were trained with $70 \%$ of the available data and tested with the remaining $30 \%$, this process was repeated using 100 monte-carlo simulations that used different, randomly selected subset of samples for training and testing. The overall percentage accuracy is reported here as ratio of successful classifications over the total number of samples, averaged over the 100 different repetitions. Each classifier was tested using the two features and three features samples shown in figure 4, as well as with the whole four extracted features (standard deviation and mean value of both centroid and bandwidth parameter). As the collection of monostatic and bistatic data was not simultaneous for these preliminary data, the classifiers were implemented separately for these two cases and their accuracy is compared in this section.

Table I and II show the accuracy obtained for monostatic and bistatic data, respectively. In both cases the $3 \mathrm{NN}$ classifier provides the best classification accuracy, up to approximately 97-98\% in the most favorable cases, followed by the BT and then NB classifier. A common trend among the three classifiers for the monostatic case is that the accuracy is at its highest when using three features and is slightly reduced when adding the fourth feature. This does not appear to happen for bistatic data, where the accuracy increases proportionally to the number of features used. This phenomenon can be related to the feature diversity effect presented in [17] for a different classification problem, i.e. the fact that different monostatic and bistatic nodes in a multistatic radar system can use different sets of features depending on situational parameters such as dwell time, signal-to-noise ratio, aspect angle to the target, and target trajectory, and that these sets of features can change dynamically in a cognitive radar paradigm. It is therefore interesting to observe this difference in these preliminary data even with a fairly limited bistatic angle $\left(20^{\circ}\right)$, and additional experimental work at other bistatic angles will be performed to further characterize this effect as part of the full paper. Confusion matrices are also shown for the case of binary tree classifier with three features, in Table III and IV for monostatic and bistatic data respectively. It is interesting to observe that the majority of the misclassification events happen between activities 1 and 2, i.e. picking up and object and sitting and standing, whereas very few mistakes are reported for the classification of the walking movement. This seems to suggest that a hierarchical process could be more suitable for indoor activities classification, with a tree of different classifiers acting on different features to discriminate different subsets of activities. A simple example of this approach was for instance presented in [21] where three Support Vector Machine classifiers were employed to discriminate between four different hand gestures. 
(a)

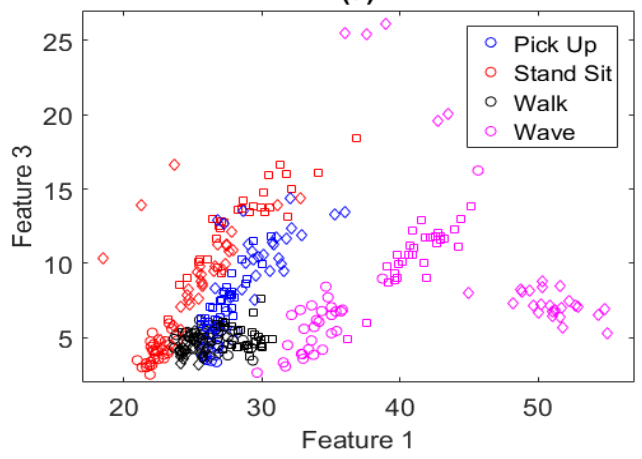

(c)

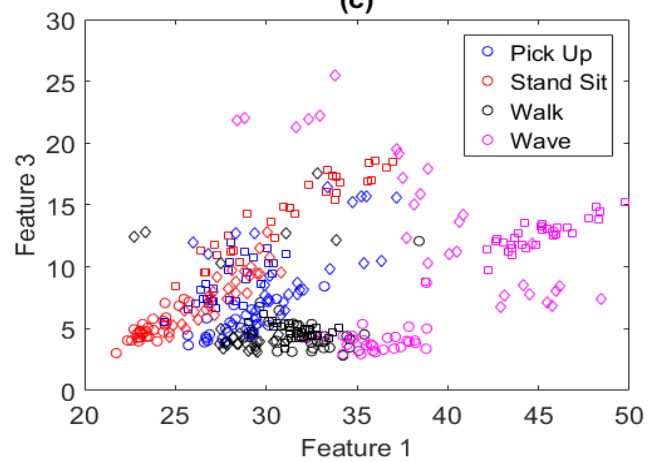

(b)

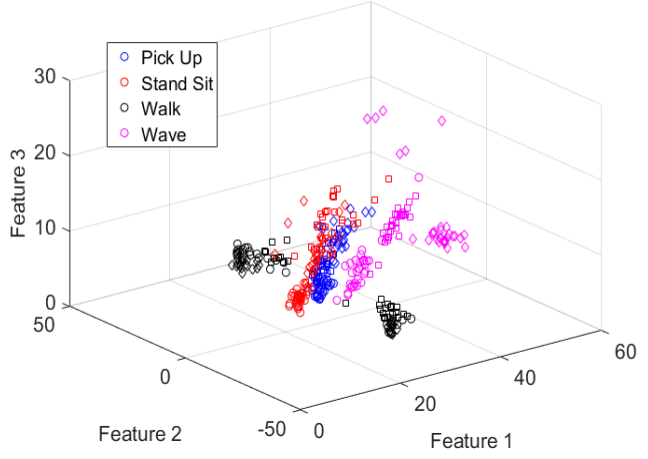

(d)

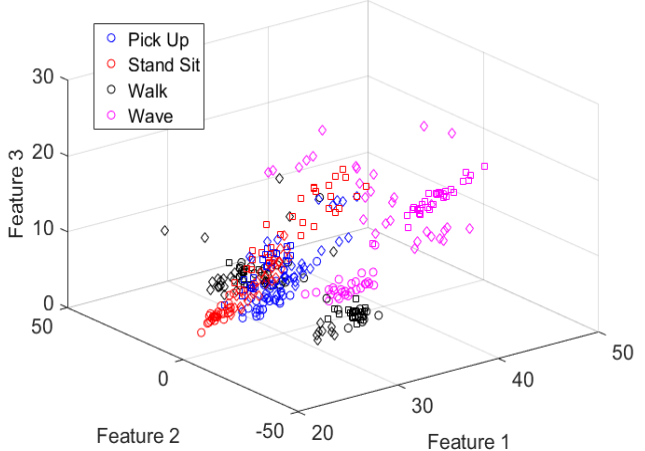

Fig. 4. Feature space plots with samples related to four actions: (a) monostatic data 2 features, (b) monostatic data 3 features, (c) bistatic data 2 features, and (d) bistatic data 3 features

TABLE I. CLASSIFICATION ACCURACY RESULTS FOR MONOSTATIC DATA

\begin{tabular}{|c|c|c|c|}
\hline Accuracy [\%] & 2 features & 3 features & 4 features \\
\hline BT & 79.5 & 90.4 & 87.3 \\
\hline NB & 71.5 & 87.7 & 86.7 \\
\hline 3NN & 89.5 & 98.3 & 97.2 \\
\hline
\end{tabular}

TABLE II. CLASSIFICATION ACCURACY RESULTS FOR BISTATIC DATA

\begin{tabular}{|c|c|c|c|}
\hline Accuracy [\%] & 2 features & 3 features & 4 features \\
\hline BT & 74.8 & 83.3 & 87.2 \\
\hline NB & 69.3 & 83.8 & 86.7 \\
\hline 3NN & 82.9 & 92.0 & 97.2 \\
\hline
\end{tabular}

TABLE III. CONFUSION MATRIX FOR MONOSTATIC DATA - BINARY TREE CLASSIFIER WITH 3 FEATURES

\begin{tabular}{|c|c|c|c|c|}
\hline $\begin{array}{c}\text { Accuracy } \\
{[\%]}\end{array}$ & A1 & A2 & A3 & A4 \\
\hline $\mathbf{A 1}$ & 75.7 & 21 & 0 & 3.3 \\
\hline $\mathbf{A 2}$ & 8 & 90.7 & 0 & 1.3 \\
\hline $\mathbf{A 3}$ & 0 & 0 & 100 & 0 \\
\hline $\mathbf{A 4}$ & 4.8 & 0 & 0 & 95.2 \\
\hline
\end{tabular}

TABLE IV. CONFUSION MATRIX FOR BISTATIC DATA - BINARY TREE CLASSIFIER WITH 3 FEATURES

\begin{tabular}{|c|c|c|c|c|}
\hline $\begin{array}{c}\text { Accuracy } \\
{[\%]}\end{array}$ & $\mathbf{A 1}$ & $\mathbf{A 2}$ & $\mathbf{A 3}$ & $\mathbf{A 4}$ \\
\hline $\mathbf{A 1}$ & 72 & 22.3 & 0 & 5.7 \\
\hline $\mathbf{A 2}$ & 19.8 & 75.7 & 0.5 & 4 \\
\hline $\mathbf{A 3}$ & 0 & 1.4 & 96.8 & 1.8 \\
\hline $\mathbf{A 4}$ & 4.6 & 6.7 & 0 & 88.7 \\
\hline
\end{tabular}

\section{CONCLUSIONS}

This paper has presented the preliminary analysis of bistatic human micro-Doppler signatures to classify indoor activities. The development of robust automatic classification approaches will benefit the deployment of systems for monitoring daily life patterns of people, especially elderly or vulnerable individuals with some form of impairment, and for detecting fall events. This simple analysis considered actions that may be mistaken for actual fall events, namely sitting down and bending to pick up objects, together with walking and waving. Accuracy up 98\% can be achieved with simple features based on the centroid and bandwidth of the micro-Doppler signatures and Nearest Neighbor classifier. It was found that the bistatic classifier performance continued to increase with added features, whereas the monostatic generally peaked at 3 feature inputs. The bistatic channel did have an overall reduction in classification performance, but this has only been shown for one bistatic angle. Further results over a series of angles will be analyzed in order to understand this phenomenon further. 
Further work will aim at collecting a larger database of activities with more actions and more subjects, and investigating the effect of the many operational parameters such as frequency, dwell time, signal-to-noise ratio, aspect angles, number of radar sensors and bistatic angles. The differences in the optimal selection of features between monostatic and bistatic data has already been highlighted in this simple analysis, and further work is needed to fully characterize and exploit this 'feature diversity'. Enhanced approaches to select subsets of features at each radar node will also be explored, for example using wrapper and filters methods proposed in the literature [5, 17], as well as hierarchical classifiers to separate different actions at different levels of the classification process.

\section{ACKNOWLEDGMENT}

The authors would like to thank A. Angelov for helping in the experimental data collection. A. Angelov was supported in this work by the UK Engineering and Physical Sciences Research Council (EPSRC) Doctoral Training Partnership Grant EP/M508056/1, through the College of Science and Engineering at the University of Glasgow. The authors acknowledge the Royal Society Newton Advance Fellowship awarded to Prof Gang Li and Prof Hugh Griffiths as support for this work.

\section{REFERENCES}

[1] V. C. Chen, D. Tahmoush, W. J. Miceli, Radar Micro-Doppler Signatures: Processing and Applications, Institution of Engineering and Technology, 2014.

[2] V. C. Chen, F. Li, S. S. Ho and H. Wechsler, 'Micro-Doppler effect in radar: phenomenon, model, and simulation study', IEEE Transactions on Aerospace and Electronic Systems, vol. 42, no. 1, pp. 2-21, Jan. 2006.

[3] D. Tahmoush, 'Review of micro-Doppler signatures', IET Radar, Sonar \& Navigation, vol. 9 (9), pp. 1140-1146, December 2015.

[4] K. Youngwook and L. Hao, "Human activity classification based on micro-Doppler signatures using a Support Vector Machine," IEEE Transactions on Geoscience and Remote Sensing, vol. 47, pp. 13281337, 2009.

[5] S. Z. Gürbüz, B. Erol, B. Çağlıyan and B. Tekeli, "Operational assessment and adaptive selection of micro-Doppler features," IET Radar, Sonar \& Navigation, vol. 9, no. 9, pp. 1196-1204, December 2015 .

[6] R. M. Narayanan and M. Zenaldin, "Radar micro-Doppler signatures of various human activities," IET Radar, Sonar \& Navigation, vol. 9, no. 9, pp. 1205-1215, December 2015.
[7] S. Björklund, H. Petersson and G. Hendeby, "Features for microDoppler based activity classification," IET Radar, Sonar \& Navigation, vol. 9, no. 9, pp. 1181-1187, December 2015.

[8] R. Ricci and A. Balleri, "Recognition of humans based on radar microDoppler shape spectrum features", IET Radar, Sonar \& Navigation, vol. 9 (9), pp. 1216-1223, December 2015.

[9] M. G. Amin, Y. D. Zhang, F. Ahmad, and K. C. D. Ho, "Radar Signal Processing for Elderly Fall Detection: The future for in-home monitoring," IEEE Signal Processing Magazine, vol. 33, no. 2. pp. 7180, 2016.

[10] B. Jokanovic, M. G. Amin, Y. D. Zhang and F. Ahmad, "Multi-window time-frequency signature reconstruction from undersampled continuouswave radar measurements for fall detection," IET Radar, Sonar \& Navigation, vol. 9, no. 2, pp. 173-183, 22015.

[11] M. G. Amin, F. Ahmad, Y. D. Zhang and B. Boashash, "Human gait recognition with cane assistive device using quadratic time-frequency distributions," IET Radar, Sonar \& Navigation, vol. 9, no. 9, pp. 12241230, December 2015.

[12] Q. Wu, Y. D. Zhang, W. Tao and M. G. Amin, "Radar-based fall detection based on Doppler time-frequency signatures for assisted living," IET Radar, Sonar \& Navigation, vol. 9, no. 2, pp. 164-172, 2 2015.

[13] C. Garripoli, M. Mercuri, P. Karsmakers, P. J. Soh, G. Crupi, G. A. E Vandenbosch, C. Pace, P. Leroux, and D. Schreurs, "Embedded DSPBased Telehealth Radar System for Remote In-Door Fall Detection," IEEE Journal of Biomedical and Health Informatics, vol. 19, no. 1. pp. 92-101, 2015

[14] B. Y. Su, K. C. Ho, M. J. Rantz, and M. Skubic, "Doppler Radar Fall Activity Detection Using the Wavelet Transform," IEEE Transactions on Biomedical Engineering, vol. 62, no. 3. pp. 865-875, 2015.

[15] F. Fioranelli, M. Ritchie, H. Griffiths, 'Aspect angle dependence and multistatic data fusion for micro-Doppler classification of armed/unarmed personnel', IET Radar, Sonar \& Navigation, vol. 9 (9), pp. 1231-1239, December 2015

[16] M. Inggs, H. Griffiths, F. Fioranelli, M. Ritchie and K. Woodbridge, "Multistatic radar: System requirements and experimental validation," 2014 International Radar Conference, Lille, 2014, pp. 1-6.

[17] F. Fioranelli, M. Ritchie, S. Gürbüz, H. Griffiths, 'Feature diversity for optimized human micro-Doppler classification using multistatic radar', IEEE Transactions on Aerospace and Electronic Systems, accepted for publication in October 2016.

[18] F. Fioranelli, M. Ritchie, H. Griffiths, 'Performance analysis of centroid and SVD features for personnel recognition using multistatic microDoppler', IEEE Geoscience and Remote Sensing Letters, vol. 13, no. 5, pp. 725-729, May 2016.

[19] F. Fioranelli, M. Ritchie, H. Griffiths, 'Centroid features for classification of armed/unarmed multiple personnel using multistatic human micro-Doppler', IET Radar, Sonar \& Navigation, accepted for publication in April 2016.

[20] T. Hastie, R. Tibshirani, J. Friedman, The Elements of Statistical Learning: Data Mining, Inference, and Prediction, Second Edition, Springer, 2009.

[21] S. Zhang, G. Li, M. Ritchie, F. Fioranelli, H. Griffiths, 'Dynamic hand gesture classification based on radar micro-Doppler signatures', presented at the 2016 CIE International Conference on Radar, Guangzhou, October 2016. 